\title{
Snapchat Streaks: How Adolescents Metagame Gamification in Social Media
}

\author{
Dayana Hristova $^{1,2}$, Joseph Dumit ${ }^{3}$, Andreas Lieberoth ${ }^{4}$ and Thomas Slunecko ${ }^{1}$ \\ ${ }^{1}$ University of Vienna, Austria \\ ${ }^{3}$ University of California, Davis, USA \\ ${ }^{4}$ Aarhus University, Denmark \\ ${ }^{2}$ dayana.hristova@univie.ac.at
}

\begin{abstract}
This paper presents strategies that Viennese adolescents use to uphold snap streaks - a gamified challenge on Snapchat inviting users to exchange at least one snap each 24 hours to keep the score. This gamification feature strongly impacts the communication practice of adolescents, both with regard to its temporality and its content. In order to secure the timed reciprocal exchange with their streak partner, adolescents would resort to so called streak snaps impersonal pictures with reduced content that are sent solely for the purpose of upholding the streak. Three partially overlapping subcategories of streak snaps are outlined: mass snaps, "good morning" or "good night" snaps, and black pictures. Their main characteristics and role in perpetuating the gamified challenge on Snapchat are discussed with regard to reciprocity and metagaming. More specifically, the impact of these metagaming strategies on adolescents' communication, as well as their relevance for social media design, is discussed in detail.
\end{abstract}

Keywords: Snapchat, Snap Streaks, gamification, social media, reciprocity, mass snaps, black snaps, metagaming

\section{Introduction}

Gamification elements have been gaining influence in a broad variety of domains $[1,2]$, their presence also shaping the face of online media [3,4]. Notably, social media have been dynamically evolving towards interface elements that could be classified as gamification. This has been particularly relevant in the case of Snapchat - a social media based on ephemeral content that allegedly enables a more private and personal communication $[5,6]$ than other platforms. In practice, users send images and videos, which immediately disappear from recipients' devices after being viewed. Recent analyses indicate that Snapchat, which has over 203 million daily active users as of 2019 [7], has become ever more heavily gamified [3] including features such as points, rewards, feedback and challenges among others.

One of the most influential features of Snapchat are "Snap Streaks": a relational score that signifies how many days in a row two users have been sending each other snaps (pictures or videos). Hristova and colleagues [3] have pointed out that streaks 
are a variation of the gamification element, i.e. points, adapted to thrive in the novel context of ephemeral social media. In other words, this form of gamification quantifies the regular online interaction of two users despite the fact that its content has disappeared. In essence, streaks are used to gamify Snapchat's "core loop" [8]: users' reciprocal exchange of snaps that is here understood as a symbolic exchange $[9,10]$. Once a streak has been established, a series of well-known psychological processes including a wish to reciprocate [11] and loss aversion [12] are likely to increase its cognitive and emotional salience. Pelaprat and Brown argue that "online life is replete with [...] exchanges through digital objects" [10] that manifest reciprocity and thereby transform individual users into constituents of social relations. As a result, this relational score has been hailed by user communities, and especially by adolescents [13] for whom "even mundane networked experiences may exert meaningful influences on well-being" [14].

Streaks also impose a temporal constraint on reciprocity: exchanging at least one snap each 24 hours. Keeping a streak alive requires ongoing effort on both partners' sides: daily attention and time devoted to it which may foster solidarity and intimacy based on the shared responsibility to reciprocate the relation [10]. Furthermore, since users cannot fake the achievement and it only takes one mistake to lose a streak, streaks can be understood as a costly signal [15]. Research has found that reciprocity decays over time [16], hence, as a gamification element, streaks will probably always be at odds with the natural processes of human interaction. The difficulty of upholding a streak together over long periods crucially adds to the perceived value of unusually long streaks creating a sense of "mutuality and pride" [10].

While the streak rules are rather simple, they have spawned a complex net of interaction practices that has not initially been foreseen. Slunecko [17] argues that any media can only be understood in the context of the actual practice with it. The specific ways of engaging psychologically and behaviorally with the predesigned set of constraints can find different expressions: e.g. re-thinking and tweaking predesigned affordances, challenging them or opening new ones. This paper presents an analysis of Viennese adolescents' social practices with streaks as they unfold in the daily involvement with others. We present three strategies adolescents use in response to the gamification element that allow them to sustain a streak and analyze these strategies in the context of reciprocity and metagaming [18].

\section{Methods}

We conducted two series of qualitative interviews at three Viennese high schools in 2018 and $2019(\mathrm{n}=26)$ as a part of a research project ${ }^{1}$ on adolescents' use of social media $[19,20]$. The analyzed data is a subset $(n=7,14-18$ years old, female $=4)$ of this larger set and includes individuals who have experience with streaks to the date of the interview or in the past. Informants were recruited through school teachers who allowed for the individual interviews (60-90 minutes) to be carried out in a separate

\footnotetext{
${ }^{1}$ Funded by a DOC-team scholarship of the Austrian Academy of Sciences.
} 
school room during their class. Participation was voluntary and a signed parent consent form was collected from each informant. Adolescents were asked about their knowledge, techniques and experience with social media using a semi-structured approach. All subjects were anonymized using pseudonyms. The interviews were coded and analyzed in Atlas.ti [21] using a modified grounded theory approach [22,23]. This method is limited in its reliance on reported experience, but allows for similar statements to be located across subjects. In this manner we were able to identify individual attitudes and strategies related to specific social media uses which were then used as the basis for iterative thematic grouping. The interviews were carried out in German and relevant quotes were translated to English for the purposes of this publication.

\section{Results: "Streak snaps"}

The practice of maintaining streaks has or had been a part of the daily routine of our informants. Although most adolescents were reluctant to admit the importance of streaks for their social life, the in-depth interviews about their daily streak practice revealed their active and emotional involvement with this gamification element. Snap streaks seem to polarize the opinions of our informants, some of whom held contradictory feelings toward their own involvement. For example, Benny $(15, \mathrm{~m})$ claimed that despite of him actively keeping streaks (as high as 400-days-long), they are "not important" for him. However, later he called the case of almost losing his streaks when banned from the phone by his parents an "emergency".

Central to our discovery of "metagaming" [18] - used here in the sense of "tricking the game" - in streaks, the interviews revealed a pool of daily strategies helping adolescents to play and to game the streak game. This paper focuses on a major set of techniques that our informants label "streak snaps" (Petra, 18, f). Elise (15, f) defines them as snaps sent "just in order to build up streaks". Since the primary goal of these snaps is to uphold a streak, the content (or the lack of it) shared through them is often not informative or exciting. Informants commonly see "streak snaps" as opposed to normal snaps that build up an authentic personal conversation that is about something. Anna (16, f) was offended by her streak partner only sending her streak snaps and never involving in a personal communication, e.g. by asking her how she is feeling. Filip $(15, \mathrm{~m})$ also prefers to snap with his friends "back-and-forth for hours rather than just for the streak". It is a wide-spread opinion among our informants that they are motivated to keep streaks just with people with whom they enjoy communicating otherwise, too. In other words, the gamified content by itself represents a diluted psychological experience of a snap that attains a new valorization in the context of numerical streaks, but only makes sense as an addition to genuine personal snaprelationship with the collaborator others.

The interviewed adolescents commonly keep streaks with several persons (often 10 and more) which, in practice, means that they receive multiple streak snaps with limited content daily. For this reason, another informant - Petra - stopped maintaining streaks. She was irritated that streaks have a negative impact on the communication with her friends: "actually, we still snap every day: what we do is sharing funny pic- 
tures and even funnier videos but when we had a streak, we partially used to take pictures of the table and send them". Petra explains that her main motivation for giving up streaks is that she would otherwise be receiving many more routine streak snaps bereft of meaning.

According to our emerging analysis, the streak snaps that our informants use daily to "game the streak game" can be grouped into the following partially overlapping themes: mass snaps, "Good morning” or "Good night” snaps, and black pictures.

\subsection{Mass snaps}

"Mass snaps" are streak snaps sent to multiple users - commonly to all streak partners, be them one's friends or just acquaintances. The strategy of creating just one snap and sending it to multiple others significantly reduces the time and effort of maintaining streaks. Mass snaps usually hold impersonal and trivial content, typically featuring pictures of shoes and the floor, food or the surroundings though their visual content is not fixed. When adolescents receive a picture that contains no personal message, it is often suspected of being a mass snap that, hence, does not require a specific answer. If one holds a streak with the sender of such a snap, the seemingly meaningless digital item is made sense of as a token of gamified exchange. As such, mass snaps are read as a sign that one further wishes to reciprocate the streak relation.

Yet, in some cases there may be ambiguity as to whether the snap is supposed to deliver information or whether it is simply a streak snap. To address this issue, some adolescents, like Nina $(16, \mathrm{f})$ and Filip, would write "streak" or "mass snap" on their snap to define the intended function of the digital item sent. This meta-comment is to establish the nature of the snap as just upholding the gamification function: the message is deprived of content apart from a self-referential remark about its intended medial function. It grants the sender's action legitimacy "so that he [the recipient] knows it's a mass snap and does not think that it's just some weird snap" (Filip). This allows for a shared meta-awareness of involving with each other in a gamified manner instead of through a content-oriented communication.

Despite the fact that our informants create and broadcast mass snaps on a daily basis, they often report perceiving them as "unnecessary" (Petra) and "pointless" (Nina). Filip also complains that mass snaps are "so not creative", but he integrates this recognition in his own practice of composing. He attempts making them more interesting through GIFs, jokes or funny stickers. His reported goal is to avoid sending mass snaps that would "bore" him if he would be the receiver. In this, Filip composes them with pride and a reciprocal sense of mutuality. Indeed, while mass snaps reduce the complexity of streak maintenance, they often turn into a stream of "unnecessary" spam for the recipient.

\subsection{Good morning/ good night snaps}

According to our informants, these snaps have a nearly fixed text content - "good morning" ("GM") and "good night" ("GN"/ "GN8") (fig. 1a,1b) - and temporality. As the names suggest, they are sent in the morning or in the evening and can be e.g. pictures taken from bed or black pictures adorned with stickers, time stamps or augment- 
ed-reality (AR) items. This more fixed rhythmic and content structure makes it easier for our informants to uphold streaks as it reduces the complexity of remembering to snap for streaks. For example, among our participants Nina prefers sending GN snaps, and Elise - GM snaps, though she shared that she occasionally also sends GN snaps in addition to her normal snap communication with her friends.

On the receiver's end, GN and GM snaps commonly build up large portions of the content users daily receive on Snapchat and can be best described as impersonal greetings, sent to multiple users, that require no direct answer. For streak holders, GM/ GN snaps are not merely anticipated but rather expected on a daily basis. Adolescents reveal knowing about others' routines and when to expect snaps from different people. For example, Elise says: "when it comes to most of my contacts, I know when they snap". Her routine is to snap in the morning but she says about her contact that "some snap only around noon". Elise also explains that "some [friends] sleep much longer than [her] on the weekend". Her statement hints at her familiarity with others' daily routines and at her awareness of their impact on streak snaps' temporal signatures. In this manner, despite their impersonality, they form the basis of reciprocal intimacy.
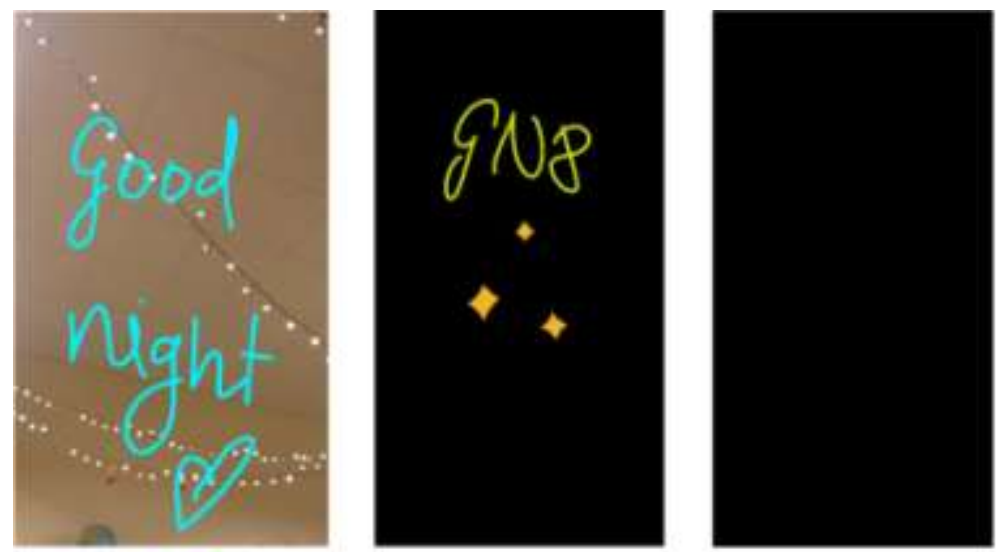

Fig. 1. Streak snaps: a) Good night snap, b) Black picture GN snap, c) Black picture

\subsection{Black snaps}

Black snaps are a specific common strategy to quickly produce and send a streak snap without visual content. Adolescents make them e.g. by covering the phone camera with a finger and then taking a picture (Nina). User can then leave the "black camera picture" (Filip) blank (fig. 1c) or add ornamentation such as text or stickers (fig. 1b). Black snaps may be sent to multiple people or to single contacts. For example, Nina who strongly dislikes black pictures says that she would send one to a single streak partner if the time was running out and the streak is endangered. Similarly, Petra states that it is only appropriate to send a black picture "within a conversation" when both users are aware that the black picture is for the purpose of streak maintenance. 
When asked about black snaps, Petra reports having used them because she had "tried everything" in order to keep her streaks. She also shares making black pictures when she had to snap for her streak but "had no good photo, or was in the bathroom, or didn't want to send a photo right now". In other words, she resorted to black pictures when she either did not have good content or did not want to share content but had to snap to uphold the gamification challenge. Black snaps commonly come across as "not creative" (Filip) and may be perceived as annoying by recipients as in the case of Nina, Filip and Petra. Adolescents report that they dislike receiving black pictures too often and hence resort to "quickly clicking [them] away" (Filip). This response is common as they are impersonal and require no specific answer. Furthermore, when the user has streaks with many others she commonly receives and has to view a wave of black pictures on a daily basis. The fact that the user continues to send and goes through these black snaps is itself form of reciprocal solidarity.

\section{Discussion}

To sum up, all of the aforementioned subtypes of streak snaps: reduce the time and effort for keeping streaks; have limited content; are impersonal and often sent to multiple others; require no specific answer; are understood as a gamified item rather than as a message; are perceived as not creative and annoying if overused. They differ in the degree to which they are unambiguously recognized as gamified items or can still be confused for a content-carrying message. These results will be discussed in the context of reciprocity, gamification and metagaming.

\subsection{Metagaming gamification}

Viewing streak snaps as the inventive response to the gamification's daily requirement to snap can be seen via Goodhart's Law [24,25], by which any index that is subject to pressure (regulatory, or here, gamification), quickly no longer functions as a meaningful sign in its original capacity. The length of a streak no longer signals the quality of a relationship between two users, but the extent to which they reciprocally care about streaks and their ability to use hacks to metagame it [18].

Streak snaps, as opposed to regular communication, are one approach to "gaming the system" by making snaps that are game-thinking and exchange placeholders rather than content carriers. Our data shows that this active response to the time pressure introduced by the gamification element is the users' attempt to reduce the time and effort needed for streak maintenance. In other words, streaks' having-to-snap (as opposed to wanting-to-snap) every day introduce a challenge that the users decide to simplify by reducing the time and effort needed for keeping the streak using various strategies that are learned and recognized within the community of practice.

Mass snaps reduce the time for streak maintenance since the user needs to invest time into making one snap (of a better or poorer quality) and send it to multiple users, rather than producing 20 individual snaps each day. The "good morning/ night" snaps (if used as mass snaps) can reduce the effort even further as the approximate time of snapping as well as the text aspect of their content are pre-defined. Both, the text and 
the time for snapping may vary slightly from day to day but it provides a rhythmic and formalized form of upholding one's streaks and performing solidarity. Black pictures are an even more significant content reduction since the visual content, possibly with the exception of text or stickers, is reduced to almost to the minimum of metadata generated.

The three types of streak snaps identified here can be seen as examples of metagaming in the sense used by Boluk \& Lemieux [18], in which players invent new ways of playing the game within the games affordances. Furthermore, individual "players" approach the streak challenge in different ways such as introducing new goals, meaning or metagames. Streak snaps are emergent techniques for daily "success in the streak game" and maintenance of reciprocal relationship by using the technology's core affordances but not exactly playing by the rules.

\subsection{Communication vs. gamification}

In the case of the aforementioned strategies, streaks' gameful nature is gaining an increasing value, potentially, at the expense of the content exchanged through snaps. In other words, the designed medium gamification, sometimes, expands at the expense of Snapchat medium's "core loop" of sending content-based messages. Petra sums up: "In my opinion, when it comes to streaks it is important that we snap and not what we snap about. That's why it doesn't matter what's in the snap - if it's personal or impersonal, or a mass snap, or you name it. Main thing is that we keep the streak". At this point, McLuhan's [26] probably most notorious argument comes to mind: "the medium is the message". In the case of streak snaps, the exchanged content is reduced to a minimum and, as a result, users' metagaming response to gamification becomes the main token of communication. Streak snaps also waive some basic communication rules, such as sending a content-specific answer in a timely manner, because the sent item contains no personal message and was probably sent to multiple others. However, while a black picture might at first occur contentless, within the context of gamified communication, similarly to backchannels (such as "uh huh" or nodding among conversation partners [27]), it signals a general desire to continue involving with the partner. The reciprocal relation of streak maintenance, hence, seems to be primarily based not on sharing content, but on the gamified exchange of digital items.

According to Pelaprat and Brown, introducing virtual rewards can potentially disrupt systems of online reciprocity and appreciation exchange between users [...]. In the case of streaks, if a person identifies that their streak partner is more interested in the point reward (the streak counter) than in the relation between the two users, the gamified exchange may start to be perceived as pointless. Some of our informants actively stopped keeping streaks with individuals who seem to care just about the streak count and not about the relation to the person. Among friends, streak snaps are sent in addition to non-gamified communication, rather than instead of it. Petra reports: "I would send mass snaps partially for the streaks, and if it is a friend of mine, I would also send the videos that I am sending now anyway. So I'd do both". While gamification can be an intriguing way to relate to others if practiced as an expansion of symbolic exchange and interpersonal relations, a stand-alone gamified exchange 
may resemble trade-like transactions and, hence, lose its relational value. As previously mentioned, some adolescents stopped keeping streaks with their friends because they perceived that a gamification-focused goal-oriented mindset had highjacked the quality of their online communication.

The imperative and shared responsibility to snap daily produces numerous metagaming items with reduced content. Although these "metagaming snaps" are placeholder signals for the continuation of gameful exchange and the mediated social relationship, they are commonly perceived as less valuable than "real snaps". However, inversely, the streak snaps "inflation" increases the value of "real snaps" that are sent between friends. Gamification, hence, paradoxically prompts more appreciation for playful (but not gameful or goal-oriented) communication among users. While our informants discussed their differentiation of the two types of snaps, social communication always remains tricky. They sometimes have trouble deciding if a snap is just a gamification item or not. In turn, they sometimes take care to make sure that their streak snaps are received as such: writing "streak" on a black picture so that it is understood as coming from an expedient need, and can serve as metacommuncation.

\subsection{Metagaming by design}

As discussed in previous literature [3,4], gamification elements are used in social media to involve users to spend more time and to generate content on their platforms. Metagaming offers an entry into understanding how users interact with and redefine the behavioral affordances offered by social media designers. This is crucial for user agency and recognizing their role in not simply "playing by the rules" but in reinventing them in interaction with the design elements offered by social media.

However, even as the players find metagaming pleasant (in reducing time and effort) or frustrating (in receiving "meaningless" snaps), the gamification designers can be said to have succeeded in intensifying involvement. Streaks can be understood as a "game format as means to achieve economies of action" [28] that attempt to steer users towards specific behaviors benefiting attention economy players. For example, Streaks encourage users to both generate content daily and to spend more time with Snapchat, thereby also increasing the app's stickiness [29]. Streaks tie series of smaller "game atoms" or "core loops" together in order to prolong "game" play [8]. As Lieberoth and collegues [30] have discussed, rather than inventing core behaviors (in this case, the sharing of ephemeral content), inserting streaks into the Snapchat interface adds a metagame structure running across instances of snapping, in order to get people to repeat certain behaviors. Adolescents may 'play' with the app in unforeseen ways, but at the same time the app still 'plays' with them - a principle coined by Slunecko [17] as 'dynamic constitution' - in that it is further expanding its penetration and 'air time' rate. In other words: even the exchange of pictures with a reduced content is in the interest of the gamification designers who are commissioned to create digital infrastructure serving global attention economy stakeholders. Metadata (e.g., the sender and recipient, the time or location of sending or viewing content) is a valuable resource for the "extraction" [28] objectives of social media companies. It is, 
hence, important to note that the metagaming described in this paper does not indicate that thereby agency is totally transferred to the side of the adolescents but instead unfolds in the interplay between users' practice and the social media companies seeking to engage their attention for as long as possible.

\section{Conclusion}

The streaks gamification feature has a strong impact on the ways our informants communicate, both with regard to the temporality and the content of their exchange. In particular, Snapchat streaks create specific temporal demands on reciprocity and these constraints prompt adolescents to reshape their peer-to-peer-communication and the manner in which they seize the gamification affordances of the app. The most striking 'solution' are the so-called streak snaps - a type of snaps exchanged to uphold streaks with less effort and time at the expense of being largely bereft of content. We would suggest for future research to observe interaction patterns over time and to investigate streaks' impact user experiences and reciprocal relations. We also encourage designers to use research on metagaming practices within social media - in their resilience, creativity and versatility as documented in this work - to create ethically sound social media affordances that enable reciprocal relations based on recognition between users [10].

\section{References}

1. Deterding, S., Dixon, D., Khaled, R., \& Nacke, L. "From game design elements to gamefulness: defining gamification." Proceedings of the 15th international academic MindTrek conference: Envisioning future media environments. ACM, (2011).

2. Hamari, J., Koivisto, J., and Sarsa, H. "Does Gamification Work?-A Literature Review of Empirical Studies on Gamification." HICSS. Vol. 14. (2014).

3. Hristova, D., Göbl, B., Jovicic, S., Slunecko, T. "The Social Media Game? How Gamification Shapes Our Social Media Engagement." In: R. Dillon (ed.), The Digital Gaming Handbook, CRC Press (In Press).

4. Lampe, C. "Gamification and social media". In S. Deterding \& S. P. Walz (Eds.), The Gameful world: Approaches, issues, applications. Cambridge, MA: MIT, 461-480. (2014).

5. Vaterlaus, J. M., Barnett, K., Roche, C., \& Young, J. A. "“Snapchat is more personal": An exploratory study on Snapchat behaviors and young adult interpersonal relationships." Computers in Human Behavior 62, pp. 594-601. (2016).

6. Utz, S., Nicole M., and Cameran K. "Snapchat elicits more jealousy than Facebook: A comparison of Snapchat and Facebook use." Cyberpsychology, Behavior, and Social Networking 18.3, pp. 141-146. (2015).

7. Zephoria, https://zephoria.com/top-10-valuable-snapchat-statistics/, last accessed 2019/11/01.

8. Elias, G., Garfield R., and Gutschera, K.R. "Characteristics of games". MIT Press, (2012).

9. Mauss, M. "The Gift: The Form and Reason for Exchange in Archaic Societies". Routledge. (1990).

10. Pelaprat, E., \& Brown, B. "Reciprocity: Understanding online social relations". First Monday, 17(10). (2012). 
11. Cialdini, R. B., Vincent, J. E., Lewis, S. K., Catalan, J., Wheeler, D., \& Darby, B. L. "Reciprocal concessions procedure for inducing compliance: The door-in-the-face technique”. Journal of Personality and Social Psychology, 31, pp. 206-215. (1975).

12. Kahneman, D. \& Tversky, A. "Advances in prospect theory: Cumulative representation of uncertainty". Journal of Risk and Uncertainty. 5 (4): pp. 297-323. (1992).

13. Déage, M. “'Don't you trust me?'Teenagers challenging friendship on Snapchat”. In ECSM 2019 6th European Conference on Social Media (p. 299). Academic Conferences and publishing limited. (2019, June).

14. Weinstein, E. "Influences of Social Media Use on adolescent Psychosocial Well-Being: 'OMG' or 'NBD'". Doctoral dissertation, Harvard Graduate School of Education. (2017).

15. Iannaccone, L. "Sacrifice and stigma: Reducing free-riding in cults, communes, and other collectives". Journal of Political Economy, 100, pp. 271-291. (1992).

16. Chuan, A., Kessler, J. B., \& Milkman, K. L. "Field study of charitable giving reveals that reciprocity decays over time". Proceedings of the National Academy of Sciences, 115(8), pp. 1766-1771. (2018).

17. Slunecko, T. „Von der Konstruktion zur dynamischen Konstitution: Beobachtungen auf der eigenen Spur". facultas. wuv/maudrich, (2008).

18. Boluk, S., and LeMieux, P. "Metagaming: Playing, competing, spectating, cheating, trading, making, and breaking videogames". Vol. 53. U of Minnesota Press, (2017).

19. Göbl, B., Hristova, D., Jovicic, S., Slunecko, T., Chevron, M. F., \& Hlavacs, H.. "Towards a More Reflective Social Media Use Through Serious Games and Co-design". In Joint International Conference on Serious Games (pp. 229-234). Springer, Cham. (2018)

20. Jovicic, S., Hristova, D., Göbl, B.: Verspielte Grenzen des Digitalen: Relationalität und Verhandlung gamifizierter $\mathrm{R}$ äume in Wiener Jugendvereinen. In: Mitteilungen der Anthropologischen Gesellschaft in Wien (MAGW). Verlag der Anthropologischen Gesellschaft (In Press)

21. Muhr, T. Atlas/ti. Berlin: Scientific Software Development. (1997).

22. Glaser, B. G., and Strauss L. A. "The discovery of grounded theory: Strategies for qualitative research". Chicago, lL: Aldine Publishing Company. (1967).

23. Clarke, A. "Situational Analysis: Grounded Theory After the Postmodern Turn". Sage Pub. (2005)

24. Griesemer, J. "Taking Goodhart's Law Meta: Gaming, Meta-Gaming, and Hacking Academic Performance Metrics”. In Biagioli, M. \& Alexandra Lippman, (ed), Metrics and Misconduct: New Ecologies of Academic Research, MIT Press. (2018).

25. Dumit, J. "Inter-pill-ation and the Instrumentalization of Compliance," afterword to New Anthropologies of Medical Compliance, special issue of Anthropology of Medicine, edited by Kalman Applbaum and Michael Oldani, v.17, n.2, February, (2010).

26. McLuhan, M. "The medium is the message". In Understanding Media: The Extensions of Man, New York: Signet. pp. 23-35, 63-7. (1964).

27. Yngve, V. "On getting a word in edgewise," Papers from the Sixth Regional Meeting of the Chicago Linguistic Society, pp. 568. (1970).

28. Zuboff, S. The age of surveillance capitalism: The fight for a human future at the new frontier of power. New York: PublicAffairs. (2019).

29. Furner, C. P., Racherla, P., and Babb, J. S. "Mobile app stickiness (MASS) and mobile interactivity: a conceptual model." The Marketing Review 14.2, pp. 163-188. (2014).

30. Lieberoth, A., Jensen, N.H., and Bredahl, T. "Selective psychological effects of nudging, gamification and rational information in converting commuters from cars to buses: A controlled field experiment." Transportation research part F: traffic psychology and behavior. (55). (2018). 